\title{
A metrological atomic force microscope for large range measurements with sub-nanometre resolution
}

\author{
Yiting Wu, Elisa Wirthmann, Ute Klöpzig, Tino Hausotte \\ Institute of Manufacturing Metrology (FMT), Friedrich-Alexander-Universität Erlangen-Nürnberg (FAU) \\ Nägelsbachstr.25, 91052 Erlangen, Germany \\ yiting.wu@fmt.fau.de
}

\begin{abstract}
Summary:
Calibration of standards is a very important task in dimensional metrology. In this paper large range measurements of pitch standards using a metrological atomic force microscope (MAFM) with combined deflection detection system, that comprises a homodyne interferometer and a tilt measuring system, are presented. The combination allows the simultaneous three-dimensional detection of tip position and cantilever bending and torsion. The combination of the MAFM sensor with the Nanopositioning and Nanomeasuring Machine allows improved quality in calibration of standards such as step height standards, pitch standards and flatness standards. This paper introduces its application for measurements of pitch standards working in contact mode (CM) and in intermitted contact mode (IM).
\end{abstract}

Keywords: metrological atomic force microscope, large range, nanomeasuring

\section{Introduction}

Scanning probe microscopy (SPM) is widely used in semiconductor, precision technology and biotechnology. As the most important member of SPMs atomic force microscopy (AFM) [1] has been used to meet the challenges for dimensional metrology in these areas. Different approaches such as optical beam deflection (OBD) systems, focus sensor detection systems and interferometric detection systems have been developed to measure the position or deflection of the cantilever [2, 3]. Measurements with a laser interferometer allow a traceability to the meter definition by means of the laser light wavelength.

A new version of a metrological laser interferometer-based AFM head has been developed at the Institute of Manufacturing Metrology (FMT). This MAFM sensor with a combined deflection detection system is not influenced by creep, hysteresis of piezo actuators, which occur in many AFM systems. The signal quality has been improved by using two wedge plates to reduce disturbing interference $[3,4]$. The new measuring system uses two tiltable plane mirrors to adjust the direction and position of a focused laser beam on the backside of the cantilever [3].

The calibration of standards is a very important task in dimensional nanometrology. For surface roughness measurements a stylus profilometer is usually used. According to DIN EN ISO 4288
[5] a minimum scan length of $400 \mu \mathrm{m}$ is required for assessment surface texture. The lateral resolution is limited by the tip radius of the stylus (typically $2 \mu \mathrm{m} \sim 5 \mu \mathrm{m}$ ). Due to the small scan ranges, the conventional AFMs are rarely used for applications in the area of conventional roughness determination. Another very important task in dimensional nanometrology is the determination of the pitch. A key factor for better statistical results of the mean pitch is the long scanning range in order to measure over a large number of grating lines [7].

The nanomeasuring machine NMM-1 developed at the IImenau University of Technology and manufactured by SIOS Meßtechnik features a reliable measurement resolution of less than $0.1 \mathrm{~nm}$ and a positioning and measuring volume of $25 \mathrm{~mm} \times 25 \mathrm{~mm} \times 5 \mathrm{~mm}$ [6]. The combination of the MAFM sensor with the NMM-1 leads to improved quality in the calibration of standards. By the integration of the MAFM in the NMM-1, large-scale measurements over a range of $25 \mathrm{~mm} \times 25 \mathrm{~mm} \times 5 \mathrm{~mm}$ with sub-nanometre resolution are realizable.

In this short paper the application of the MAFM sensor in different working modes for large range measurements of pitch standards with sub-nanometre resolution is presented.

\section{Calibration}

In order to perform measurements, the signals of the MAFM sensor must be calibrated. In CM, the sensitivity of bending signal must be deter- 
mined. In IM, oscillation amplitude of the bending signal must be calibrated. The characteristic curves of bending and oscillation amplitude sampled by the analogue digital converter of the NMM-1 are shown in Fig. 1 and Fig. 2. The working range, set point (needed for the force control by measurements in constant-force mode) and coefficients of the characteristic line (used for the calculation of the distance between the cantilever tip and the measured object) can be determined from the approach / retract curves, which also feature the typical snap-in and snap-out.

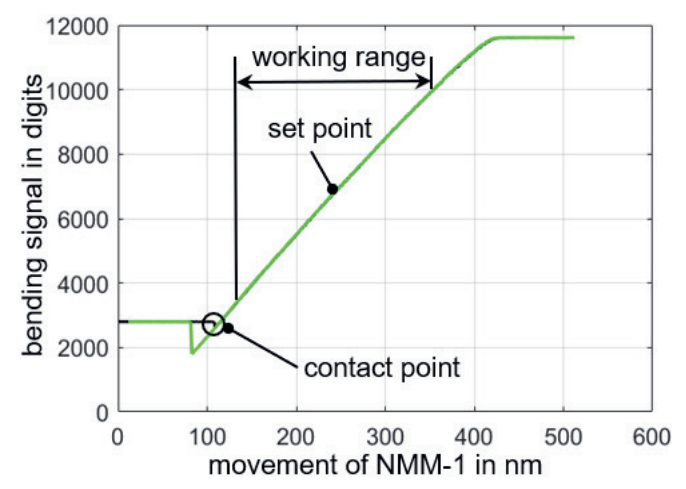

Fig. 1 Characteristic curve for bending

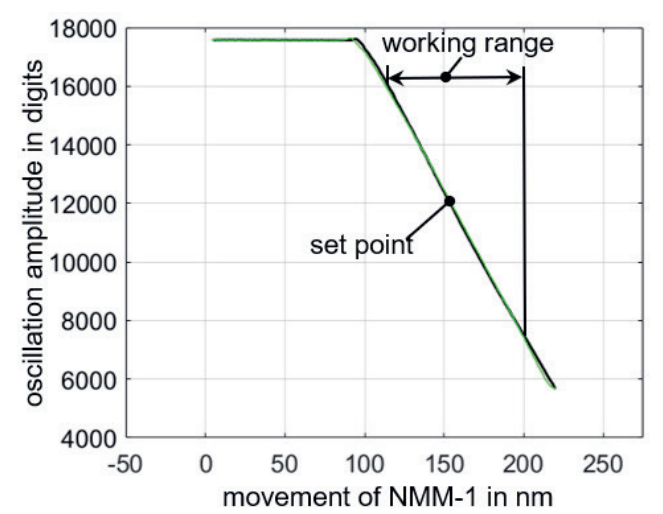

Fig. 2 Characteristic curve for oscillation amplitude

\section{Application}

The grating set TGZ series with a period of $(3 \pm 0.01) \mu \mathrm{m}$ from company NT-MDT were used in the $\mathrm{CM}$ and IM measurements. The measurements with scan length of $600 \mu \mathrm{m}$ are conducted forwards (f) and backwards (b) perpendicular to the gratings for 10 times. When measuring in IM, the profile height (z-direction) is given as the difference between the calibrated amplitude signal and the z-axis position data of the NMM-1. For measurements in CM, the profile height (z-direction) is calculated as the difference between the calibrated bending signal and the z-axis position data of the NMM-1. The mean pitch was calculated using the Fourier transform method $[3,7]$. Table 1 shows the determined mean pitch values $(m)$ and standard deviations (s) of TGZ1 with a step height of $(21.4 \pm 1.5) \mathrm{nm}$ and of TGZ2 with a step height of $(108 \pm 2) \mathrm{nm}$ by forward and backward measurements. The deviations between the nominal values and measured values for the pitch are within the uncertainty of the nominal values. The deviation between $\mathrm{CM}$ and IM was less than $50 \mathrm{pm}$. Measurements at different positions showed, that the local deviations also play a role for the measurements results.

Tab. 1: Results for pitch on gratings

\begin{tabular}{|c|c|c|c|c|}
\hline \multirow{2}{*}{ standard } & \multicolumn{2}{|c|}{ forward } & \multicolumn{2}{c|}{ backward } \\
\cline { 2 - 5 } & $m$ in nm & $s$ in pm & $m$ in nm & $s$ in pm \\
\hline \multicolumn{5}{|c|}{ CM } \\
\hline TGZ1 & 3000.058 & 82.09 & 3000.053 & 84.03 \\
\hline TGZ2 & 3000.011 & 16.85 & 3000.056 & 74.16 \\
\hline \multicolumn{5}{|c|}{ IM } \\
\hline TGZ1 & 3000.030 & 38.5 & 3000.038 & 65.3 \\
\hline TGZ2 & 3000.037 & 41.3 & 3000.023 & 24.3 \\
\hline
\end{tabular}

\section{References}

[1] H.-U. Danzebrink, T. Dziomba, M. Xu, A. Pidduck, R. Leach, A. Yaccot, L. Koenders, Scanning Probe Microscopy, Scanning Electron Microscopy and Critical Dimension: Nanometrology Status and Future Needs within Europe, Nanometrology Discussion Papers Co-Nanomet, 55-57 (2009); ISBN 978-0-9566809-0-7

[2] A. Yacoot, L. Koenders, Recent developments in dimensional nanometrology using AFMS Measurement Science and Technology, 122001 (2011); doi: 10.1088/0957-0233/22/12/122001

[3] Y. T. Wu, E. Wirthmann, U. Klöpzig, T. Hausotte, Investigation of a metrological atomic force microscope system with a combined cantilever position, bending and torsion detection system, SMSI (2020); doi: 10.5162/SMSI2020/C6.3

[4] F.G. Balzer, T. Hausotte, N. Dorozhovets, E. Manske, G. Jäger, Tactile 3D microprobe system with exchangeable styli, Measurement Science and Technology, 094018 (2011); doi: 10.1088/0957-0233/22/9/094018

[5] DIN EN ISO 4288:1998-04, Geometrische Produktspezifikation (GPS) - Oberflächenbeschaffenheit: Tastschnittverfahren - Regeln und Verfahren für die Beurteilung der Oberflächenbeschaffenheit (ISO 4288:1996)

[6] T. Hausotte, Nanopositionier- und Nanomessmaschinen - Geräte für hochpräzise makro- bis nanoskalige Oberflächen- und Koordinatenmessungen, IImenau: Techn. Univ. Habilitationsschrift, Pro Business (2011); URL: https://www.fmt.tf.fau.de/forschung/publikationen/ habilitationsschriften/\#collapse_0

[7] G. Dai, L. Koenders, F. Pohlenz, T. Dziomba und H.-U. Danzebrink, Accurate and traceable calibration of one-dimensional gratings, Measurement Science and Technology, (2005); doi: 10.1088/0957-0233/16/06/001 